\title{
Testing a Storytelling Tool for Digital Humanities
}

\author{
Fabio Pittarello \\ Università Ca' Foscari Venezia \\ Via Torino 155 - Venezia, Italia \\ pitt@unive.it
}

\begin{abstract}
This work presents an evaluation of ToBoA-3D, a social web platform for the annotation of $3 D$ models and the creation of stories. ToBoA-3D was evaluated through a pilot study realized in a real educational context, the 2014 edition of the Fall School in Digital Humanities, held at the Università Ca' Foscari Venezia in collaboration with the École Polytechnique Fédérale de Lausanne (EPFL). The tool was presented during the School's lectures and then used by the students for creating stories during the labs' activities. The results of the study were collected through direct observation and a questionnaire. The answers evidenced positive feedbacks for the core features of the platform and led to define an initial set of guidelines for its educational use.
\end{abstract}

Keywords: annotation; cultural heritage; digital humanities; education; pilot study; storytelling; web3d

\section{INTRODUCTION}

The availability of $3 \mathrm{D}$ representations for scholars is a great opportunity to support the processes of teaching and learning, especially for those disciplines that are deeply involved in the study of objects that have a 3D shape. A further exploitation of 3D objects can be obtained by their annotation that permits, for example, to search annotated content across a set of different 3D worlds, overcoming the navigation mechanisms provided by the authors of each model. ToBoA-3D [1] is a social web platform that permits to exploit the educational potential of annotated 3D worlds. It can be used for collaborative annotation, navigation and search of 3D worlds; the users can even add new 3D environments to the web repository and share them with the other users. The latest evolution of the platform [2] introduces the possibility to create educational stories on the top of the annotated worlds and to share them on the web. While ToBoA-3D can be personalized for different knowledge domains, so far the development has been focused on art and architecture. In this work we describe how ToBoA3D was tested in a real educational context, a necessary step for evidencing its points of strength and weaknesses and designing more extensive educational experiences. The occasion for the pilot study came from the Fall School in Digital Humanities, held in Venice in October 2014, in the context of the collaboration between the Università $\mathrm{Ca}^{\prime}$ Foscari Venezia and the EPFL (École Polytechnique Fédérale de Lausanne). Digital Humanities are an area of research and teaching at the intersection of computing and humanistic disciplines. This area combines the methodologies of the traditional humanities with tools and methods provided by computer science. The faculty of the Venice Fall School was compliant with this intersection, being composed by art and architecture historians but also computer scientists, which transmitted to the students complementary knowledge about the School's educational themes. The School was focused on the Venetian Renaissance and included class lessons, visits to historical sites and labs. About 20 students, mainly $\mathrm{PhD}$ students in Humanities, were selected for participating to the School, programmed for a full week. During this week a group of students had the opportunity to learn how to use ToBoA-3D and to apply this knowledge to the School's themes. The results of the experience were collected through direct observation and a questionnaire and led to define a set of guidelines for the future use of ToBoA-3D.

\section{RELATED WORKS}

The related literature encompasses different fields, from annotation of 3D worlds to storytelling. Because of space limits we'll give only some hints, addressing to [1] [2] for further references. As far as annotation is concerned, there are different proposals for adding high-level semantics to the components of 3D environments, based on specifications such as MPEG-7, Collada or X3D. Most proposals use annotations referred to taxonomies or ontologies, but there are also systems that permit to use free tags [1]. The latter ones offer a more expressive and informal approach suited also to common people. Their benefits for cultural heritage have already been underlined in [3]. Annotation is however a first step towards a more advanced use of 3D worlds. Scopigno et al. [4] underline that the greater challenge for digital technologies is the creation of tools that use 3D models for supporting cultural heritage research. We claim that the introduction of storytelling for annotated 3D worlds can bring further advantages for researchers and pupils. Computer-enhanced storytelling represents an evolution of traditional storytelling. It takes advantage of technology for creating and delivering stories, but also for designing and managing new narrative models and proposing new relations between the narration, the reader and the context. The analysis of narratology, the science that studies the structure of the story, has greatly helped the building of models and architectures for interactive storytelling [5]. Several studies have demonstrated the usefulness of storytelling for educational experiences [6]. In the cultural heritage domain storytelling is used for engaging students during the learning process, associating the narration to multimedia components [7], real scenes [8], augmented [9] and virtual reality [10]. While other tools offer repositories of assets for speeding the creation of stories, ToBoA-3D fully exploits the potential of collaboration and knowledge sharing. 


\section{The ToBoA-3D PlatForm}

Each user can contribute to the ToBoA-3D platform in different fashions, uploading 3D models, annotating the components that define the 3D environments or building narrations. All the 3D environments belonging to the platform can be explored both using a first-person navigation style or querying the system for retrieving interesting objects and places contained inside of them. Altough users are not required to perform all the types of activities, a typical session with ToBoA-3D includes a mix of them. As far as the creation of narrations is concerned, the author creates a story starting from a list of personal bookmarks, corresponding to visited $3 \mathrm{D}$ viewpoints related to annotated objects and locations, and selects an ordered set of them for defining the stages of a linear story. Each stage is then associated to multimedia content that will be automatically played during the narration. Additional content, such as textual descriptions, images and links to web resources, can be included. The interface for listening to stories is displayed in Fig.1. The story is automatically played synchronizing the delivery of the information associated to each stage with the automatic navigation to the associated 3D locations. If the story is narrated across different environments belonging to the platform, the system takes care of downloading automatically the required environment. The story can be stopped at any time, for allowing the listener to focus on details, exploring the 3D view or accessing the associated hypermedia. Stories can be navigated also selecting the single stage from the list available on the lower panel of the interface. Two key features of ToBoA-3D are that the results of all the activities are shared and that their authors are easily identifiable. The first feature enables all the users to take advantage of the work done by the other members of the community (e.g., upload of 3D models, annotations), avoiding to start from scratch for authoring. The second feature offers interesting scenarios for research and teaching. For example ToBoA-3D permits students to annotate a set of 3D architecture components after a lesson focused on classical orders and then the teacher to check the annotations made by each student, marked with the student's ID. Further details about the ToBoA-3D functionalities are available in [2].

\section{ToBoA-3D AT THE FALL SCHOOL}

The goal of the Fall School was to investigate, through a set of coordinated lectures and visits to Venetian palaces and collections, new ways of visualizing the evolution of architecture and artwork display. The School was focused in particular on investigating the evolution of the Grimani Palace in Venice and of the artwork collection contained in its main room, the so-called Tribuna. During the preparatory work we discussed with the other organizers how to present and use the annotation platform during the School's activities. A skilled 3D modeler created a simplified model of the Grimani Palace and its Tribuna that was used as the scenario for an educational narration built with ToBoA-3D. The narration was created with the contribution of Cristiano Guerneri, one of the architecture historians involved in the School. It was organized as a self-paced linear story, guiding the students with a virtual camera through the locations of the palace. The first part of the School included lectures held by art historians and focused on the main School's theme, but also talks related to the use of new technologies for cultural heritage, among which ToBoA-3D. The last two days of the school were dedicated to technological labs, held in parallel for small groups of students. For this reason, only four students out of twenty had the chance to attend the ToBoA-3D lab. In spite of the low number of students, the results were interesting. The initial phase of the ToBoA-3D lab was dedicated to a tutorial illustrating the different features of the platform. The students were then invited to try the techniques acquired on some test 3D environments prepared for the School. Finally the students listened to the introductory story by the art historian that ended with the presentation of two tasks to accomplish:

- the creation of a narration describing a tour through the rooms of the palace, enriched with the snapshots taken during the visit to the real building;

- the creation of a a story related to an hypothesis of reallocation of the artworks of the Tribuna Grimani, which are currently placed in a different site.

The students interpreted both the themes proposed, altough with some simplifications due to time constraints. The first story was an humorous interpretation where one of the students played the part of a Venetian nobleman and guided the listeners through the rooms of the palace (see Fig.1 on the left). The second story was a more serious narration, focused on the hypothesis of reallocation of the artworks in the Tribuna Grimani (see Fig.1 on the right; the red dots in the 3D scene represent the original position of the artworks).

\section{Results of The Pilot Study}

The four students were $\mathrm{PhD}$ candidates representatives of different research domains: visual arts and architecture (two students), computer science and interaction design, literature. While we should not consider this study as exhaustive, their answers have a great value for identifying the points of weakness and strength of the platform from different facets. Only two of them (the computer scientist and one of the art historians) had a fair knowledge of modeling techniques and interactive 3D environments. None of them had previous experience related to the annotation of $3 \mathrm{D}$ worlds. The results of the pilot study were collected through discussions with students and a final questionnaire, composed of closed and open questions, and articulated in 6 sections focused on annotation, search, storytelling, sharing, workflow and usability/engagement. We obtained positive results for most of the features of ToBoA-3D. While we don't have the space to analyze the single results, we underline how the pilot study led to define more precisely the profile of the students interested in using this platform and the set of tasks that should define a complete experience. These results will be useful for designing a more advanced study or more extensive and complete educational experiences based ToBoA-3D: 

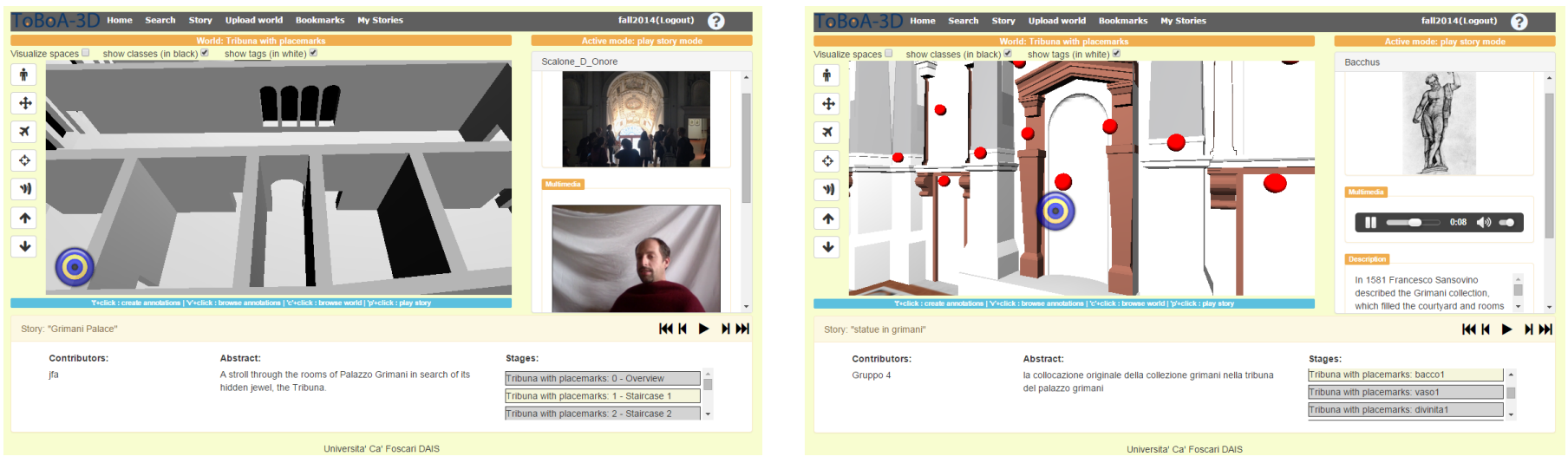

Fig. 1. The two stories created by the students at the Digital Humanities Venice Fall School

- design educational experiences for students interested in visual representation; the study revealed that ToBoA-3D resulted more appealing to students with a background focused on visual representation rather than on literature; we suggest, as a complementary guideline, that for longer term experiences such as a master course, the introduction of a basic 3D modeling course would be useful for giving a basic knowledge of 3D representation and attracting students with different cultural backgrounds;

- include search and information sharing among the activities of the educational experience; in the pilot study students were shown the functionalities related to search and information sharing and declared their interest for them; however, starting also from the observations of the students, we estimate that it would be interesting to include specific tasks focused on these activities in the structure of the educational experience, proposing for example to search an annotated set of 3D worlds in relation to a given goal or to ask a group students to annotate individually the same set of objects and then check the annotations of their fellows for identifying different point of view; probably these activities would augment the awareness of the potential of these techniques and would stimulate their application to individual research;

- include knowledge checking as part of the educational experience; while the educational experience proposed in this experience included a set of goals defined by a teacher, for time constraints it was not possible to fulfill all the teacher's requests and to check the results; a full educational experience should include a final check by the teacher of the individual work and a feedback to students.

Other suggestions came from the feedback related to the ToBoA-3D functionalities. While we obtained positive judgments for the core features of ToBoA-3D, most of the students complained about the quality of the 3D models, realized under heavy time constraints. This is an issue that we'll take into account for the future educational experiences. The students suggested improvements to the platform as well. While the students with an humanistic background focused more on content and structure, suggesting for example the possibility to create stories with branching structures, the computer science student focused on interaction design issues, suggesting ways to refine the interface or adding additional functionalities. While the positive findings encourage us to propose the use of the platform in real educational contexts, the future development will take care of all the points of weaknesses underlined by the users and improve further its features.

\section{ACKNOWLEDGMENTS}

Thanks to Ivano Gatto for all the contributions given to the development of the ToBoA-3D platform. I acknowledge Frédéric Kaplan and Isabella Di Lenardo (EPFL), which coorganized the Fall School in Digital Humanites, and Cristiano Guarneri (Università IUAV), which gave a great contribution for the educational story for the students.

\section{REFERENCES}

[1] F. Pittarello and I. Gatto, “ToBoA-3D: an architecture for managing topdown and bottom-up annotated $3 \mathrm{~d}$ objects and spaces on the web," in Proc. of Web3D'11, 2011, pp. 57-65.

[2] I. Gatto and F. Pittarello, "Creating web3d educational stories from crowdsourced annotations," Journal of Visual Languages \& Computing, vol. 25 , no. 6 , pp. 808-817, 2014.

[3] J. Trant, "Exploring the potential for social tagging and folksonomy in art museums: Proof of concept," New Review of Hypermedia and Multimedia, vol. 12, no. 1, pp. 83-105, 2006.

[4] R. Scopigno, M. Callieri, P. Cignoni, M. Corsini, M. Dellepiane, F. Ponchio, and G. Ranzuglia, "3D Models for Cultural Heritage: Beyond Plain Visualization,” Computer, vol. 44, no. 7, pp. 48-55, Jul. 2011.

[5] M. Cavazza and D. Pizzi, "Narratology for interactive storytelling: A critical introduction," in Proc. of TIDSE'06. Berlin, Heidelberg: Springer-Verlag, 2006, pp. 72-83.

[6] J. Ohler, Digital Storytelling in the Classroom: New Media Pathways to Literacy, Learning, and Creativity. Corwin Press, 2008.

[7] K. Kwiatek and M. Woolner, "Let me understand the poetry: Embedding interactive storytelling within panoramic virtual environments," in Proc. of EVA'10, 2010, pp. 199-205.

[8] J. Halloran, E. Hornecker, G. Fitzpatrick, M. Weal, D. Millard, D. Michaelides, D. Cruickshank, and D. De Roure, "The literacy fieldtrip: using ubicomp to support children's creative writing," in Proc. of IDC' '06, 2006, pp. 17-24.

[9] Z. Zhou, A. D. Cheok, J. Pan, and Y. Li, "An interactive 3d exploration narrative interface for storytelling," in Proc. of IDC '04. New York, NY, USA: ACM, 2004, pp. 155-156.

[10] S. Mystakidis, N. Lambropoulos, H. M. Fardoun, and D. M. Alghazzawi, "Playful blended digital storytelling in $3 \mathrm{~d}$ immersive elearning environments: A cost effective early literacy motivation method," in Proc. of IDEE '14. New York, NY, USA: ACM, 2014, pp. 97-101. 